International Journal of Applied Mathematics

Volume 31 No. $6 \quad 2018,797-803$

ISSN: 1311-1728 (printed version); ISSN: 1314-8060 (on-line version)

doi: http://dx.doi.org/10.12732/ijam.v31i6.8

\title{
ON THE RAINBOW NEIGHBOURHOOD NUMBER OF MYCIELSKI TYPE GRAPHS
}

\author{
N.K. Sudev ${ }^{1}$, C. Susanth ${ }^{2}$, S.J. Kalayathankal ${ }^{3}$ \\ ${ }^{1}$ Department of Mathematics \\ CHRIST (Deemed to be University) \\ Bengaluru - 560029, INDIA \\ 2 Department of Mathematics \\ Vidya Academy of Science \& Technology \\ Thalakkottukara, Thrissur - 680501, INDIA \\ ${ }^{3}$ Department of Mathematics \\ Kuriakose Elias College \\ Mannanam, Kottayam - 686561, INDIA
}

\begin{abstract}
A rainbow neighbourhood of a graph $G$ is the closed neighbourhood $N[v]$ of a vertex $v \in V(G)$ which contains at least one colored vertex of each color in the chromatic coloring $\mathcal{C}$ of $G$. Let $G$ be a graph with a chromatic coloring $\mathcal{C}$ defined on it. The number of vertices in $G$ yielding rainbow neighbourhoods is called the rainbow neighbourhood number of the graph $G$, denoted by $r_{\chi}(G)$. In this paper, we discuss the rainbow neighbourhood number of the Mycielski type graphs of graphs.
\end{abstract}

AMS Subject Classification: $05 \mathrm{C} 15,05 \mathrm{C} 75$

Key Words: color classes, rainbow neighbourhood, rainbow neighbourhood number

\section{Introduction}

For general terminology in graph theory, we refer the reader to $[2,8]$. For further concepts on graph coloring, refer to $[1,3]$. Unless stated otherwise, the

Received: June 18, 2018

(C) 2018 Academic Publications

$\S_{\text {Correspondence author }}$ 
graphs mentioned in this paper are simple, connected, finite and undirected graphs.

A coloring of a graph $G$ is an assignment of colors to its elements (vertices and/or edges) subject to certain conditions. Many practical and real life situations paved paths to different graph coloring problems.

By graph coloring, we mean an assignment of colors to the vertices of the graph under consideration so that no two adjacent vertices have the same color. A color class of a graph is the set of all its vertices having the same color. Note that the color classes in a graph $G$ are independent sets in $G$. A $k$-coloring of a graph $G$ uses $k$ colors to color its vertices, by partitioning the vertex set $V$ into $k$ color classes. The minimum value of $k$ for which $G$ admits a $k$-coloring is called the chromatic number of $G$, denoted by $\chi(G)$.

\subsection{Rainbow Neighbourhoods in a Graph}

A rainbow neighbourhood of a graph $G$, which admits a chromatic coloring $\mathcal{C}$, is the closed neighbourhood $N[v]$ of a vertex $v \in V(G)$ which contains at least one vertex from each color class of $\mathcal{C}$ (see [4]). The rainbow neighbourhood number of the graph $G$, denoted by $r_{\chi}(G)$, is the number of vertices in $G$ belonging to some rainbow neighbourhoods in $G$ (see [4]).

The rainbow neighbourhood number of certain fundamental graph classes have been determined in $[4,6,7]$. Some of the relevant results in these paper are as follows:

(i) For $n \geq 1, r_{\chi}\left(P_{n}\right)=n$.

(ii) For $n \geq 3, r_{\chi}\left(C_{n}\right)= \begin{cases}3, & \text { if } n \text { is odd, } \\ n, & \text { if } n \text { is even. }\end{cases}$

(iii) For $n \geq 1, r_{\chi}\left(K_{n}\right)=n$.

It is proved in [4] that for any graph $G$ on $n$ vertices, $\chi(G) \leq r_{\chi}(G) \leq n$ and for any bipartite graph $G$ on $n$ vertices, $r_{\chi}(G)=n$. Also, for any graph $G$, the graph $G^{\prime}=K_{1}+G$ has $r_{\chi}\left(G^{\prime}\right)=1+r_{\chi}(G)$ (See [4]).

\subsection{Mycielski Graph of a Graph}

The Mycielski graph of a given graph $G$ with vertex set $V(G)=\left\{v_{1}, v_{2}, v_{3}, \ldots, v_{n}\right\}$ is the graph obtained by applying the following steps:

1. Corresponding to each vertex $v_{i}$ in $V(G)$, introduce a new vertex $u_{i}$ and 
let $U=\left\{u_{i}: 1 \leq i \leq n\right\}$. Add edges from each vertex $u_{i}$ of $U$ to the vertex $v_{j}$ if $v_{i} v_{j} \in E(G)$.

2. Take another vertex $u$ and add edges from $u$ to all vertices in $U$.

The new graph thus obtained is called the Mycielski graph of $G$ and is denoted by $\mu(G)$ (see [5]).

The following figures (in Figure 1) illustrate the Mycielski graphs or Mycielskian of a path and a cycle.

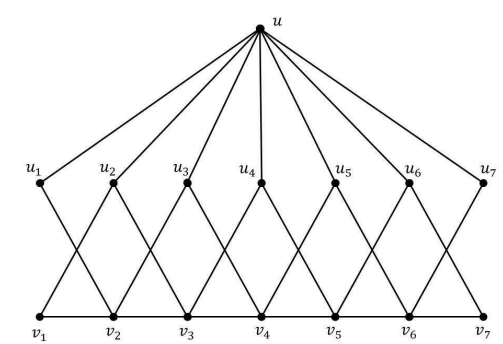

(a) Mycielski graph of $P_{7}$.

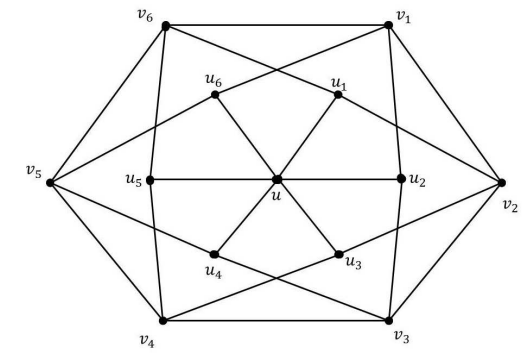

(b) Mycielski graph of $C_{6}$.

Figure 1

In this paper, we investigate the rainbow neighbourhood number of certain graph classes.

\section{Rainbow neighbourhood Number of Mycielski Graphs}

In this discussion, we follow the convention that a coloring $\left(c_{1}, c_{2}, c_{3}\right.$, $\left.\ldots, c_{\chi(G)}\right)$ is used in such a way that the color $c_{1}$ is assigned to maximum possible number of vertices, then the color $c_{2}$ is given to maximum possible number of vertices remaining uncolored and proceeding like this, at the final step, the remaining uncolored vertices are given color $c_{\ell}$. This convention may be called the rainbow neighbourhood convention (see [4]).

\subsection{New Results}

Theorem 1. For any graph $G, r_{\chi}(\mu(G))=r_{\chi}(G)+1$.

Proof. Let $V=\left\{v_{1}, v_{2}, \ldots, v_{n}\right\}$ be the vertex set of $G$. Let $U=\left\{u_{1}, u_{2}, \ldots\right.$, $\left.u_{n}\right\}$ be the set of newly introduced vertices such that $u_{i}$ corresponds to the 
vertex $v_{i}$ such that $u_{i}$ is adjacent to $v_{j}$ and $v_{k}$ provided $v_{j}$ and $v_{k}$ are adjacent to $v_{i}$. Take another vertex $u \neq u_{i}, u \neq v_{i}$ for $1 \leq i \leq k$, and add edges from $u$ to $u_{i}$ for all $i$. Let $\mathcal{C}=\left\{c_{1}, c_{2}, \ldots, c_{k}\right\}$ be the chromatic coloring of the graph $G$. Since $u_{i}$ is not adjacent to $v_{i}, u_{i}$ and $v_{i}$ can have the same color. Since $u$ is adjacent to all $u_{i}$, no color in $\mathcal{C}$ can be assigned to the vertex $u$. Therefore, we have to consider a new color, say $c_{k+1}$ for the vertex $u$. Since no vertices in $V$ is adjacent to the vertex $u$ in $\mu(G)$, it is clear that the vertices in $V$ will not be in the rainbow neighbourhood of $\mu(G)$. If $v_{i}$ is a vertex in the rainbow neighbourhood of $G$, it can be observed that the corresponding vertex $u_{i}$ will be in a rainbow neighbourhood of $\mu(G)$. Since $u_{i}$ is adjacent to the vertices in all color classes in $\mathcal{C}$ and is adjacent to the vertex $u$ having color $c_{k+1}$. Therefore, every vertex in $U$ belongs to some rainbow neighbourhoods of $\mu(G)$. Moreover the vertex $u$ is adjacent to all vertices of $U$, which belong to different color classes in $\mathcal{C}$ and hence belongs to some rainbow neighbourhoods of $\mu(G)$. Therefore, $r_{\chi}(\mu(G))=r_{\chi}(G)+1$.

Corollary 2. The rainbow neighbourhood number of $\mu\left(P_{n}\right), r_{\chi}\left(\mu\left(P_{n}\right)\right)=$ $n+1$.

Corollary 3. The rainbow neighbourhood number of $\mu\left(C_{n}\right)$,

$$
r_{\chi}\left(\mu\left(C_{n}\right)\right)=\left\{\begin{array}{ll}
n+1 & \text { if } n \text { is even } \\
4 & \text { if } n \text { is odd }
\end{array} .\right.
$$

\section{Crib graph of a graph}

The crib graph of a given graph $G$ with vertex set $V(G)=\left\{v_{1}, v_{2}, v_{3}\right.$, $\left.\ldots, v_{n}\right\}$ is the graph obtained by applying the following steps:

1. Corresponding to each vertex $v_{i}$ in $V(G)$, introduce a new vertex $u_{i}$ and let $U=\left\{u_{i}: 1 \leq i \leq n\right\}$. Add edges from each vertex $u_{i}$ of $U$ to the vertex $v_{j}$ if $v_{i} v_{j} \in E(G)$.

2. Take another vertex $u$ and add edges from $u$ to all vertices in both $U$ and $V$.

The new graph thus obtained is called the crib graph of $G$ and is denoted by $c(G)$.

The following Figure 2 illustrates the crib graph or crib of a path. 


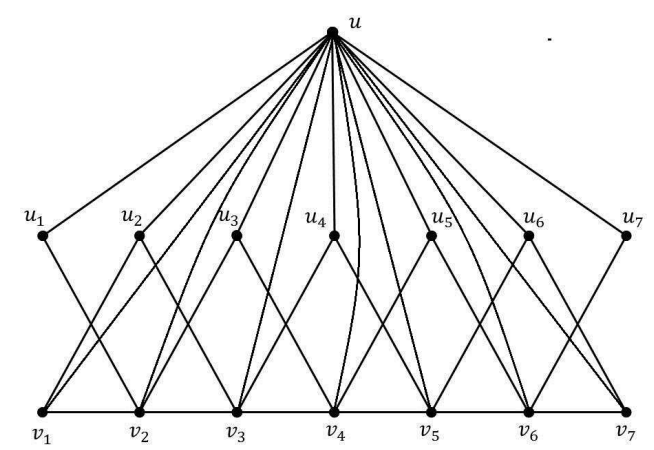

Figure 2: Crib graph of $P_{7}$

Theorem 4. For any graph $G, r_{\chi}(c(G))=2 r_{\chi}(G)+1$.

Proof. Let $V=\left\{v_{1}, v_{2}, \ldots, v_{n}\right\}$ be the vertex set of $G$. Let $U=\left\{u_{1}, u_{2}, \ldots\right.$, $\left.u_{n}\right\}$ be the set of newly introduced vertices such that $u_{i}$ corresponds to the vertex $v_{i}$ such that $u_{i}$ is adjacent to $v_{j}$ and $v_{k}$ provided $v_{j}$ and $v_{k}$ are adjacent to $v_{i}$. Take another vertex $u \neq u_{i}, u \neq v_{i}$ for $1 \leq i \leq k$, and add edjes from $u$ to $u_{i}$ for all $i$. Let $\mathcal{C}=\left\{c_{1}, c_{2}, \ldots, c_{k}\right\}$ be the chromatic coloring of the graph $G$. Since $u_{i}$ is not adjacent to $v_{i}, u_{i}$ and $v_{i}$ can have the same color. Since $u$ is adjacent to all $u_{i}$, no color in $\mathcal{C}$ can be assigned to the vertex $u$. Therefore, we have to consider a new color, say $c_{k+1}$ for the vertex $u$.

Note that all vertices in $V$ is adjacent to the vertex $u$ in $c(G)$. Therefore, $u$ belongs to a rainbow neighbourhood in $c(G)$. Also, $c_{k+1}$ is the only color which is not assigned to $V$ in $G$, we note that all vertices in $V$ belongs to some rainbow neighbourhood of $c(G)$. As mentioned in the proof of the previous theorem, the corresponding vertex $u_{i}$ will be in a rainbow neighbourhood of $c(G)$. Since $u_{i}$ is adjacent to the vertices in all color classes in $\mathcal{C}$ and is adjacent to the vertex $u$ having color $c_{k+1}$. Therefore, every vertex in $U$ belongs to some rainbow neighbourhoods of $c(G)$. Moreover, the vertex $u$ is adjacent to all vertices of $U$, which belong to different color classes in $\mathcal{C}$ and hence belongs to some rainbow neighbourhoods of $c(G)$. Therefore, $r_{\chi}(c(G))=2 r_{\chi}(G)+1$. 


\section{Snare graph of a graph}

The snare graph of a given graph $G$ with vertex set $V(G)=\left\{v_{1}, v_{2}, v_{3}\right.$, $\left.\ldots, v_{n}\right\}$ is the graph obtained by applying the following steps:

1. Corresponding to each vertex $v_{i}$ in $V(G)$, introduce two new vertices $u_{i}$ and $w_{i}$ and let $U=\left\{u_{i}: 1 \leq i \leq n\right\}$ and $W=\left\{u_{j}: 1 \leq j \leq n\right\}$. Add edges from each vertex $u_{i}$ and $w_{i}$ of $U$ and $W$ to the vertex $v_{j}$ if $v_{i} v_{j} \in E(G)$.

2. Take another vertex $u$ and add edges from $u$ to all vertices in both $U$ and $W$.

The new graph thus obtained is called the snare graph of $G$ and is denoted by $s(G)$.

The following Figure 3 illustrates the snare graph or snare graph of a path.

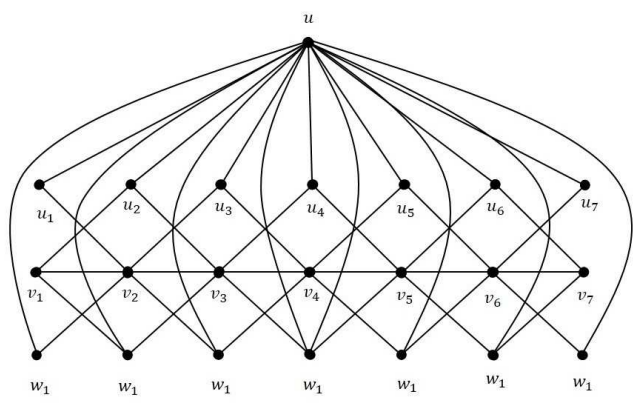

Figure 3: Snare graph of $P_{7}$

Theorem 5. For any graph $G, r_{\chi}(s(G))=2 r_{\chi}(G)+1$.

Proof. As explained in the previous proof, we observe that no vertices of $V$ belong to the rainbow neighbourhood of $s(G)$. But all the vertices in $U$ and $W$ and the vertex $u$ are in some rainbow neighbourhoods of $s(G)$ completing the proof.

\section{Conclusion}

In this paper, we have discussed a special type of graph coloring and a related parameter called rainbow neighbourhood number of certain Mycielskian type 
of graphs. This study can be extended to other derived graphs, graph products and several interesting graph classes. The rainbow neighbourhood number of power graphs can also be studied in detail.

\section{References}

[1] G. Chartrand and P. Zhang, Chromatic Graph Theory, CRC Press, Boca Raton (2009).

[2] F. Harary, Graph Theory, New Age International, New Delhi (2001).

[3] T.R. Jensen and B. Toft, Graph Coloring Problems, John Wiley \& Sons (1995).

[4] J. Kok, N.K. Sudev, M.K. Jamil, Rainbow neighbourhoods of graphs, Proy. J. Math., (2018), In Press.

[5] W. Lin, J. Wu, P.C.B. Lam, G. Gu, Several parameters of generalized Mycielskians, Discrete Appl. Math., 154, No 8 (2006), 1173-1182; DOI:10.1016/j.dam.2005.11.001.

[6] N.K. Sudev, C. Susanth, S.J. Kalayathankal, J. Kok, A note on the rainbow neighbourhood number of graphs, Nat. Acd. Sci. Lett., Online First (2018); DOI: $10.1007 / \mathrm{s} 40009-018-0702-6$.

[7] N.K. Sudev, C. Susanth, S.J. Kalayathankal, J. Kok, Some new results on the rainbow neighbourhood number of graphs, Nat. Acd. Sci. Lett., Online First (2018); DOI: 10.1007/s40009-018-0740-0.

[8] D.B. West, Introduction to Graph Theory, Pearson Education Inc., Delhi (2001). 
